\title{
In Situ Polymerized pCBT Composites with Aligned Carbon Nanotube Buckypaper: Structure and Properties
}

\author{
Zhongrui Li," Rebekah Downes, Zhiyong Liang
}

High-performance carbon-nanotube-based thermoplastic composites are synthesized through in situ polymerization of cyclic butylene terephthalate (pCBT) on aligned carbon nanotube buckypaper. The initial results show that the buckypaper/pCBT composites have a tensile strength of approximately $600 \mathrm{MPa}$ and a modulus of $96 \mathrm{GPa}$ due to the aligned nanotubes in the composite acting as a framework and the in situ polymerization resulting in increased interfacial interactions between the PCBT chains and nanotubes. The buckypaper/pCBT composites also demonstrate significantly improved thermal $\left(70 \mathrm{~W} \mathrm{~m}^{-1}{ }^{\circ} \mathrm{C}^{-1}\right)$ and electric conductivities $\left(526 \mathrm{~S} \mathrm{~cm}^{-1}\right.$ ), as well as thermoelectric power (Seebeck coefficient of $\left.64 \mu \mathrm{V}^{\circ} \mathrm{C}^{-1}\right)$. With low mass density, these combined excellent multi-properties indicate that the composites can act as multifunctional materials in numerous applications.

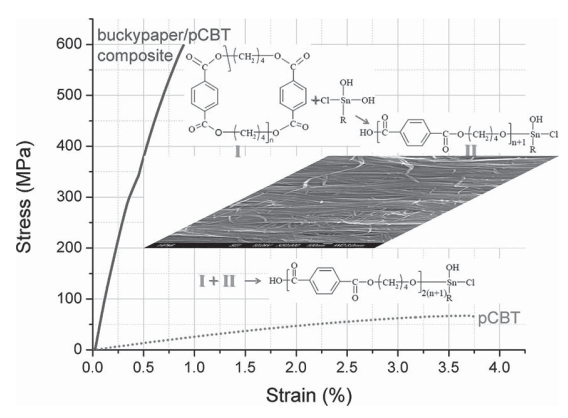

\section{Introduction}

Thermoplastics offer many advantages over their thermosetting counterparts in terms of fabrication cycle, damage tolerance, surface finish, shelf-life, and recyclability (low scrap). Due to their long molecular chains, conventional thermoplastics have much higher melt viscosity values $\left(\approx 10^{2}-10^{4} \mathrm{~Pa} s\right)$ and high melting temperature, which often becomes one of the main issues when using thermoplastics as polymer matrices. This issue can be avoided by using cyclic oligomers to develop thermoplastic resins that can be polymerized reactively like thermosetting resins but behave like thermoplastics. Cyclic oligomers have many special attributes such as low processing viscosity and rapid processibility (polymerization in a short

Dr. Z. Li

Electron Microbeam Analysis Laboratory (EMAL),

University of Michigan, MI 48109, USA

E-mail: zhongrui@umich.edu

R. Downes, Prof. Z. Liang

High Performance Materials Institute (HPMI),

Florida State University, FL 32310, USA timescale, less than $10 \mathrm{~min}$ ). The low dynamic viscosity (ca. $1 \mathrm{~Pa} \mathrm{~s}$ ) allows for use as a resin for impregnating longfiber reinforced composites.

One of the cyclic oligomers that has received much attention in the areas of polymer blends, ${ }^{[1]}$ micro-, ${ }^{[2]}$ and nanocomposites, ${ }^{\left[{ }^{[3]}\right.}$ is cyclic butylene terephthalate (CBT). ${ }^{[4]} \mathrm{CBT}$ has a water-like processing viscosity as low as $0.02 \mathrm{~Pa} \mathrm{~s}$, and can be transformed into linear high molecular weight polybutylene terephthalate (PBT) via entropically-driven ring-opening polymerization in a short timescale. ${ }^{[5]}$ CBT represents a new chemical route to semi-crystalline thermoplastic polybutylene terephthalate. Their ring-opening polymerization, associated with crystallization, may be completed below the melting temperature of the resulting polybutylene terephthalate (referred to as pCBT). ${ }^{[6]}$ The pCBT thermoplastic resin has a high melting point $\left(>230^{\circ} \mathrm{C}\right)$ and physical performance similar to that of other commercially available PBT resins. pCBT may have great importance for "all-PBT" composites. The thermoplastic nature of PCBT may provide a low-capital route to a new family of pCBT-based recyclable materials made using a range of plastic processing technologies. ${ }^{[7]}$ 
<smiles>CCOc1ccc(OCCCOC(C)(C)C)cc1</smiles>

I<smiles>[R][Si](O)(O)Cl</smiles>

II

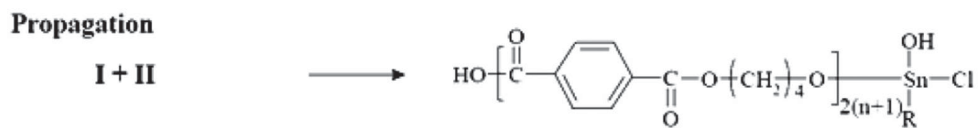

Scheme 1. A schematic of the mechanism for the ring-opening polymerization of cyclic PBT via activated linears. After adding an initiator, it will polymerize into the better known polybutyleneterephthalate (PBT). Depending on the initiation, it will polymerize into a macrocyclic or into linear PBT. [9]

However, thermoplastics still suffer from poor tensile performance compared to thermosets. The invention of carbon nanotubes (CNTs) is a game-changing revolution in the fabrication of high performance composites thanks to their unique combination of extremely strong tensile strength and high electrical and thermal conductivities, as well as their other excellent properties. ${ }^{[8]}$ With a small diameter, a high aspect-ratio, and a large surface area, carbon nanotubes are viewed as an ideal filler for polymers to enhance their performance. In this work, we report on the synthesis of high performance buckypaper/ pCBT composites through an in situ polymerization approach. As a network assembly of carbon nanotubes, buckypapers offer opportunities for promising real-world structural applications. CBT can melt completely at about $150{ }^{\circ} \mathrm{C}$ to produce a low-viscosity fluid that is ideal for wetting carbon nanotube networks and reinforcement, thereby enabling the development of composites that were previously impossible when working with high-viscosity commercial PBT. In this approach, with the assistance of a Sn-containing catalyst, CBT oligomers start ring opening and in-situ polymerization on the wall surfaces of carbon nanotubes at the melting temperature since this process happens in the timeframe of manufacturing. The in situ polymerization mechanism of CBT with ring opening is illustrated in Scheme $1 .^{[9]}$

\section{Experimental Section}

Cyclic butylene terephthalate pellets (CBT-160) were purchased from Cyclics Corporation, where a self-containing Sn catalyst was designed for the ring-opening polymerization. The process parameters, including time, temperature, catalyst percentage, and stirring, are crucial to the kinetics of the ring-opening and polycondensation reactions, which determine the molecular weight, melting point, and mechanical properties (toughness above all) of the obtained engineering polymer. ${ }^{[10]} \mathrm{CBT}-160$ can be processed from $190^{\circ} \mathrm{C}$ to $250^{\circ} \mathrm{C}$. Usually, the higher temperature results in a shorter cure and a shorter pot life. It is known that the best mechanical properties are achieved if the polymerization happens above the melt temperature of PBT $\left(230^{\circ} \mathrm{C}\right)$. Toughness can be maximized by rapid cooling to develop more amorphous content. Apart from rapid cooling, various copolymerization and chemical modification routes are also possible. ${ }^{[11,12]}$ Steel shim stock was used as a mold to produce the pCBT films. The CBT powder was filled into the mold slot and then subjected to a pressure of $13.8 \mathrm{MPa}$ at $250{ }^{\circ} \mathrm{C}$ for $15 \mathrm{~min}$, then allowed to cool to room temperature. The resulting polymer is chemically identical to PBT with a different molecular weight and crystalline fraction, so it was designated as PCBT here for clarity.

To synthesize high performance buckypaper/pCBT composites, CBT pellets were first dissolved in chloroform solution, buckypaper strips $(1.2 \mathrm{~cm} \times 5 \mathrm{~cm})$ were stretched in order to align the carbon nanotubes. The carbon nanotube sheets were purchased from Nanocomp Technologies Inc. (Concord, New Hampshire, USA). The lengths of the multi-walled nanotubes were about $1 \mathrm{~mm}$. The strips were subjected to a consistent and slow uniaxial strain under $\approx 65{ }^{\circ} \mathrm{C}$ until $50 \%$ elongation was achieved (i.e., the post-stretched sheet was $50 \%$ longer than the pre-stretched one). The aligned buckpaper was impregnated with CBT/chloroform solution $\left(0.5 \mathrm{mg} \mathrm{mL}^{-1}\right)$ for $12 \mathrm{~h}$, and then dried at $150{ }^{\circ} \mathrm{C}$ in air. The CBT-coated buckypaper strips were sandwiched with two pieces of steel shim and smoothed with a roller, and then heated at $250{ }^{\circ} \mathrm{C}$ under a pressure of approximately $14 \mathrm{MPa}$ for $15 \mathrm{~min}$ (using a 10 ton hydraulic Carver press, Wabash, Indiana, USA). Finally they were cooled by turning off the heater and opening the hot press. The rapid cooling may help create an amorphous phase of pCBT. The final composites were named based on the weight of buckypaper, i.e., $x \mathrm{BpCBT}$, where $x$ was the weight percentage of buckypaper in the composite.

The surface and cross-sectional morphologies of the composite samples were investigated using SEM. SEM measurements were performed on a field emission scanning electron microscope (JEOL $7401 \mathrm{~F}$ ) using a voltage of $10 \mathrm{kV}$. Small- and wide-angle X-ray scattering (SAXS and WAXS) measurements were performed using a Bruker NanoSTAR system with an Incoatec IlS microfocus X-ray source operating at $45 \mathrm{kV}$ and $650 \mu \mathrm{A}$. The primary beam was collimated with cross-coupled Gobel mirrors and a pinhole of $0.1 \mathrm{~mm}$ in diameter, providing a $\mathrm{Cu} \mathrm{K} \alpha$ radiation beam $(\lambda=0.154 \mathrm{~nm})$ with a beam size of about $0.15 \mathrm{~mm}$ at full width half maximum

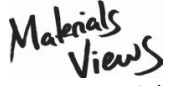

www.MaterialsViews.com
Macromol. Chem. Phys. 2015, 216, 292-300

(c) 2014 WILEY-VCH Verlag GmbH \& Co. KGaA, Weinheim

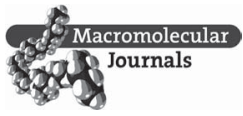


(FWHM) at the sample position. The small-angle scattering intensity was measured on a two-dimensional multiwire HiSTAR detector. The wide-angle diffraction pattern was captured on a Fuji Photo Film image plate, and read with a Fuji FLA-7000 scanner. Fourier transform infrared (FTIR) spectra of PCBT and its buckypaper composites were obtained in transmission mode on a Nicolet Magna IR-860 FTIR spectrometer. The samples were mixed with $\mathrm{KBr}$ powder, pressed into a $1 \mathrm{~cm}$ disc (ca. $0.5 \mathrm{wt} \% \mathrm{CBT}$ ), and placed on the sample holder. A background absorption spectrum was taken before each run and subtracted from the sample spectrum. All spectra were recorded from $400-4000 \mathrm{~cm}^{-1}$. A total of 32 scans at a resolution of $2 \mathrm{~cm}^{-1}$ were averaged.

The stiffness (modulus) change as a function of the temperature was studied with dynamic mechanical analysis (DMA). Rectangular shaped samples that were $25 \mathrm{~mm}$ long and $5 \mathrm{~mm}$ wide were mounted in a large tension clamp on a TA Instruments DMA Q-800. For each sample, the temperature was ramped from $50{ }^{\circ} \mathrm{C}$ to $300{ }^{\circ} \mathrm{C}$, at a $3^{\circ} \mathrm{C} \mathrm{min}^{-1}$ heating rate. An oscillating $(1 \mathrm{~Hz})$ force with an amplitude of $0.1 \mathrm{~N}$ was applied to the sample in the tensile mode, and the deformation of the sample and the lag between the applied force signal and the sample's response were measured. The loss-to-storage $\left(E^{\prime \prime} / E^{\prime}\right)$ modulus ratio is the damping $(\tan \delta)$, which is a measure of force (energy) dissipation. The tensile properties of the composite samples were measured using a Shimadzu AGS-J materials testing system (Kyoto, Japan) at room temperature $\left(23 \pm 2{ }^{\circ} \mathrm{C}\right)$ and $40 \pm 5 \%$ relative humidity,

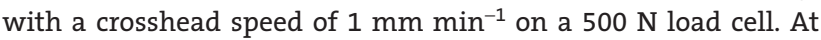
least three specimens of each composite type were tested to ensure reproducibility. The electrical and thermal conductivities of the buckypaper/pCBT composites were measured along the stretching direction using a physical property measurement system (PPMS, Quantum Design). The samples, typically $\approx 0.05 \mathrm{~mm}$ thick, were cut into $10 \mathrm{~mm}$ (stretching direction) $\times 2 \mathrm{~mm}$ strips, and the probe distance was about $5 \mathrm{~mm}$. A resistive heater and a temperature sensor were attached to one end of a sample through a metal lead using thermally conductive silver epoxy, while the other end was attached to a cold foot and a second temperature sensor. At a given power setting of the heater, the temperature difference between the two sensors was used to calculate the in-plane thermal conductance. High vacuum and radiation shields were used to minimize heat loss from the heater. The electrical conductivity was measured afterward with the same two-probe contacts at the same temperature.

\section{Results and Discussion}

\subsection{X-ray Scattering, FTIR, and DSC Characterization of Buckypaper/pCBT Composites Synthesized through an In Situ Polymerization Approach}

Both small- and wide-angle X-ray scattering were utilized to study the crystalline structure and layer distance (d-spacing) of pCBT and buckypaper/pCBT composites synthesized through the in situ polymerization approach. The 2D scattering patterns of these materials are displayed in Figure 1. Typically, the features (peaks and shoulders) in SAXS arise from either domains or lattice-like structures,

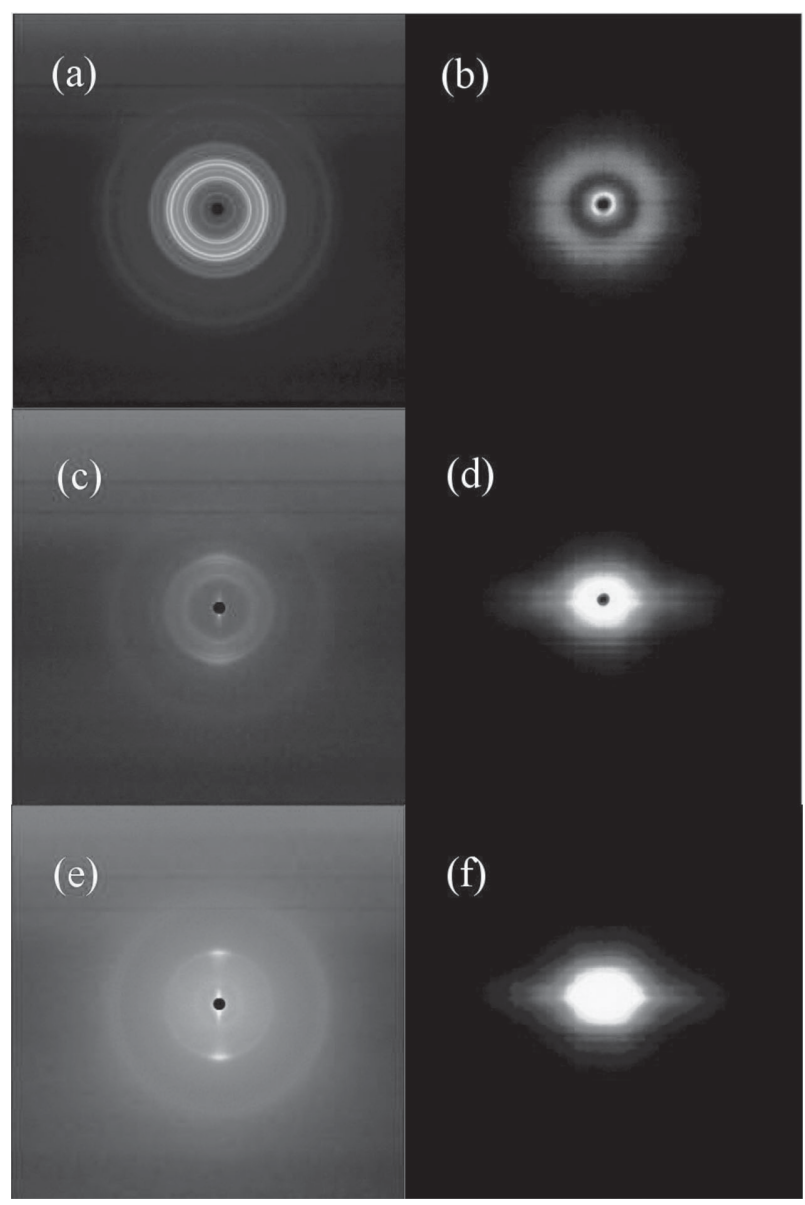

Figure 1. The $2 \mathrm{D}$ pattern of wide (left column) and small (right column) angle X-ray scattering from the pCBT $(a, b), 64 B p C B T(c, d)$ and stretched buckypaper (e,f) samples.

and the characteristic length associated with the domain (domain size) and lattice (d-spacing among planes) can be estimated from the peak positions using $2 \pi / q$. For the pCBT sample, it may form a layered structure; two characteristic lengths were approximately $57 \mathrm{~nm}$ and $13 \mathrm{~nm}$. The WAXS graph suggests that pCBT is in its alpha crystalline form with a triclinic unit cell. Characteristic (2 theta) peaks of this cell are theoretically the following: $9.1^{\circ}$ (001), $16.1^{\circ}(101), 17.2^{\circ}$ (010), $20.5^{\circ}$ (102), $23.2^{\circ}(100), 25.4^{\circ}$ (021), $27.4^{\circ}(003), 30.7^{\circ}(113), 39.3^{\circ}(105) \cdot{ }^{[13,14]}$ All of these peaks are well resolved in the scatter (Figure $2 \mathrm{~b}$ ). The short bump under the diffraction peaks between $10^{\circ}$ and $35^{\circ}$ is due to an amorphous halo. ${ }^{[15,16]}$ This suggests that, in the presence of a suitable catalyst, the CBT oligomers follow the ring-opening and polymerization process and form a linear polymer.

In contrast to the isotropic distribution of the halo in PCBT, the WAXS/SAXS patterns of the aligned buckypaper and their composites are anisotropic. The presence of arcs is a characteristic feature of X-ray patterns from a uniaxial oriented sample. The orientation degree of the 

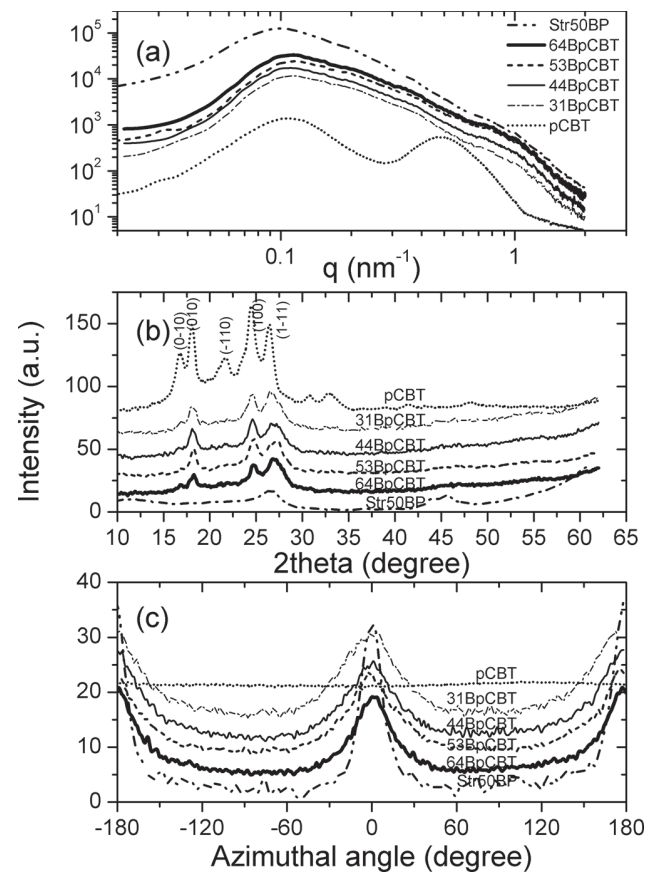

Figure 2. a,b) The SAXS (a) and WAXS (b) profiles of pCBT and 50\%-stretched buckypaper (Str5OBp) and their composites xBpCBT. c) The azimuthal scan at the graphitic diffraction (ooz) peak $26^{\circ} \pm 5^{\circ}$.

respective scattering planes is directly reflected in the azimuthal width of the arcs. The relative intensity along the azimuthal, $(I(\phi))$, at $2 \theta$ is related to the orientation distribution function (ODF) of the scattering planes. When approximating the ODF as a Legendre polynomial series in $\cos \phi$, the Herman's orientation parameter $S_{\mathrm{d}}$ (sometimes referred to as P2) is the second moment average of the ODF and is expressed as shown in Equation 1 and 2:

$$
\begin{aligned}
& S_{d}=\frac{3\left\langle\cos ^{2} \phi\right\rangle-1}{2} \\
& \left\langle\cos ^{2} \phi\right\rangle=\frac{\int_{0}^{\pi} I(\phi) \sin \phi \cos ^{2} \phi d \phi}{\int_{0}^{\pi} I(\phi) \sin \phi d \phi}
\end{aligned}
$$

For uniaxial orientation, $S_{d}$ assumes values from -0.5 to 1. A value of -0.5 reflects perfect alignment in the plane perpendicular to the uniaxial direction; 0 reflects random orientation, and 1 reflects alignment along the uniaxial direction. ${ }^{[17]}$ The 50\%-stretched buckypaper shows narrow distributed arcs in both WAXS and SAXS patterns due to the high alignment of carbon nanotubes in the buckypaper, which was also indicated by the narrow width of the azimuthal scan at the graphitic <002> crystal plane scattering around $26.7^{\circ}$. The aligned fraction was estimated to be $0.86 \pm 0.05$ for the $50 \%$ stretched buckypaper. For the composites, the intensity of the diffraction peak of the graphitic layer at $26.7^{\circ}$ decreases with increasing PCBT content. Additionally, the arc width (full width at half maximum) of the peak in the azimuthal scan increases from $18.5^{\circ}$ in $50 \%$-stretched buckypaper to $41.3^{\circ}$ in the sample 31BpCBT, suggesting that a high PCBT content does affect the alignment of carbon nanotubes in the composite. This might be due to the fact that the molten CBT or $\mathrm{pCBT}$ has a very low viscosity $(\approx 0.02 \mathrm{~Pa}$ s, waterlike) which causes CNTs/bundles to slide under pressure, accordingly changing the orientation of the CNTs.

The interfacial interaction between pCBT and carbon nanotubes was examined using transmission FTIR. The pCBT polymer exhibits similar FTIR feature peaks to the CBT oligomer (Figure 3). The most intense bands of the spectra of pCBT at room temperature were located at $3054 \mathrm{~cm}^{-1}$ (CH aromatic), 2961, 2897, $2876 \mathrm{~cm}^{-1}\left(\mathrm{CH}_{2}\right)$, $1709 \mathrm{~cm}^{-1}$ (C=O), $1419 \mathrm{~cm}^{-1}$ (aromatic ring), $1268 \mathrm{~cm}^{-1}$ $(\mathrm{C}(=\mathrm{O})-\mathrm{O}), 1104 \mathrm{~cm}^{-1}\left(\mathrm{O}-\mathrm{CH}_{2}\right), 1018 \mathrm{~cm}^{-1}$ (aromatic ring), and $728 \mathrm{~cm}^{-1}$ ( $\mathrm{CH}$ aromatic ring), in good agreement with the literature. ${ }^{[18,19]}$ In the buckypaper spectrum, bands were found at 1629, 2857, 2919, and $2953 \mathrm{~cm}^{-1}$, along with a bump at around $3444 \mathrm{~cm}^{-1}$. After the CBT in situ polymerization on the buckypaper, some pCBT-related peaks, such as $499,807,921,1167,1327,1949,3068,3416$, 3547 , and $3622 \mathrm{~cm}^{-1}$, disappear or are significantly weakened. This might suggest that the pCBT polymer chain binds on the carbon nanotube surfaces, which constrains certain bond vibrations.

The differential scanning calorimetry thermograms for the buckypaper/pCBT compositions, as well as the pCBT sample under heating and subsequent cooling, are displayed in Figure $4 \mathrm{a}$ and $4 \mathrm{~b}$, respectively. Multiple endotherm peaks can be observed in all of these samples

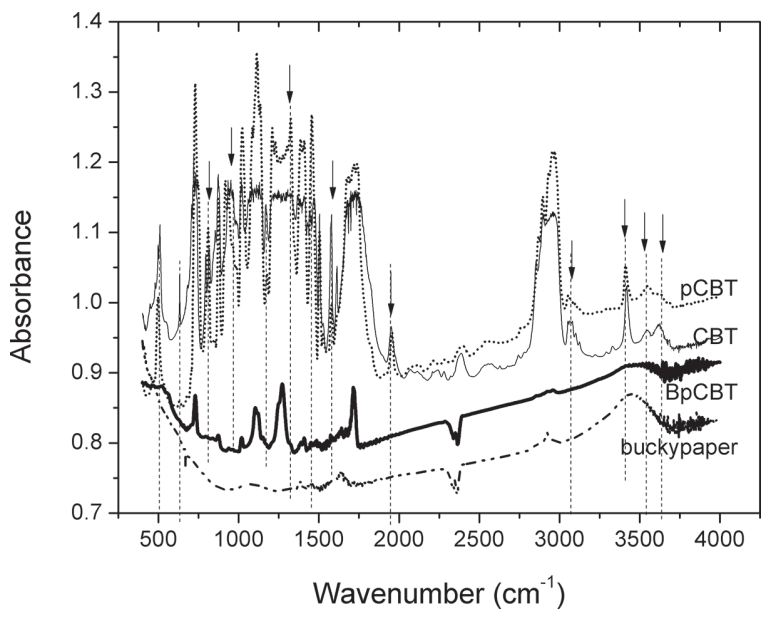

Figure 3. The FTIR spectra of CBT, pCBT, buckypaper/pCBT (BpCBT) composite, and buckypaper. The arrows mark where the vibrations disappeared or are significantly weakened in the composite due to the interaction between carbon nanotubes and PCBT chains.

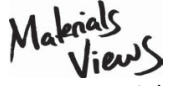

www.MaterialsViews.com 


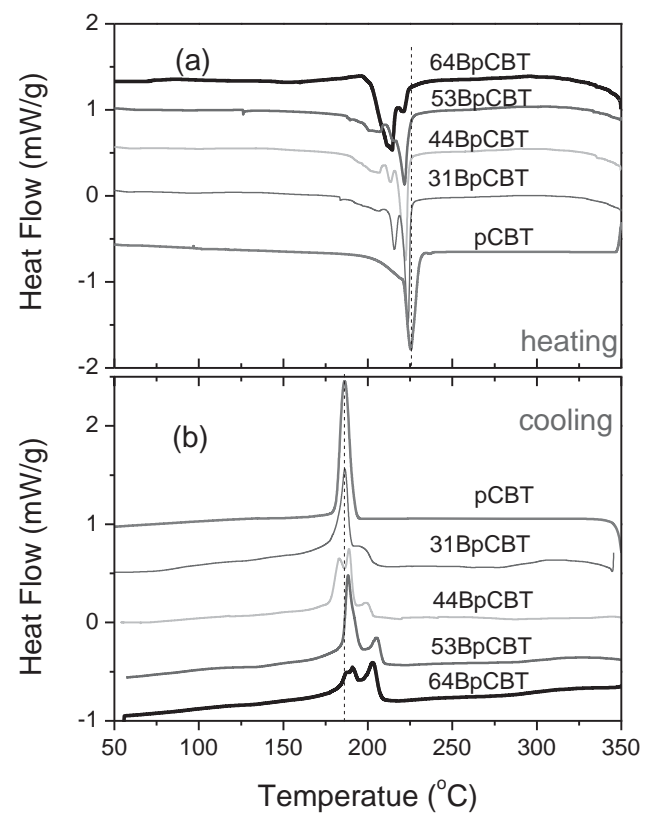

Figure 4. The DSC thermograms for buckypaper/pCBT compositions measured during melting (a) and cooling (b).

during the heating scan, which is a characteristic phenomenon for PCBT obtained from the in situ polymerization of CBT. ${ }^{[20,21]}$ The multiple melting is well known and also shown by a modulated DSC technique unequivocally. ${ }^{[22]}$ The multiple melting peaks of the thermoplastics might suggest the existence of two or more groups of crystals with different morphologies caused by remelting-recrystallization processes during DSC scanning, the lower one representing the melting of imperfect or smaller/thinner crystals, and the higher one representing bigger crystallites. ${ }^{[23,24]}$ As the content of carbon nanotubes increases, the areas of the melting peaks of the composites located at low temperature increase while those at the higher temperature gradually decrease and shift to a lower temperature. During the cooling scan, only one crystallization peak was observed for the PCBT sample, indicating a single-mode distribution in the crystallization size during the cooling process. The crystallization peak temperature of pCBT is $185.7^{\circ} \mathrm{C}$. However, with increasing carbon nanotube content in the composite, multiple crystallization peaks appear and the crystallization temperatures increase. Similar observations have been reported by others. ${ }^{[25,26]}$

\subsection{Electrical and Thermal Conductivities of the pCBT/CNT Composites Synthesized through an In Situ Polymerization Approach}

Carbon nanotubes have excellent phonon transportability with an experimentally measured individual-nanotube thermal conductivity of $3000 \mathrm{~W} \mathrm{~m}{ }^{-1}{ }^{\circ} \mathrm{C}^{-1}$, [27] which can be used to improve the thermal conductivities of polymeric composites. ${ }^{[28,29]}$ As can be seen from Figure 5 a, from -268 to $52{ }^{\circ} \mathrm{C}$, the thermal conductivity of our 50\%-stretched buckypaper (Str50BP) sample increased smoothly as the temperature increased, and displayed a temperature dependence similar to those of random ${ }^{[30]}$ and aligned single-wall carbon nanotube buckypapers. ${ }^{[31]}$ At room temperature, the thermal conductivity of our 50\%-stretched buckypaper sample was around $90 \mathrm{~W} \mathrm{~m}^{-1}{ }^{\circ} \mathrm{C}^{-1}$ within an order of magnitude of that of diamond or graphite. ${ }^{[32]}$ The fact that the better-aligned nanotubes exhibited higher $\mathrm{k}$ values than graphite might be due to the low dimensionality of the nanotubes suppressing the Umklapp processes. ${ }^{[33]}$ As expected, the thermal conductivity of the stretched buckypaper/pCBT composite decreased as the buckypaper content decreased; when 85\% pCBT was added into the buckypaper, the thermal conductivity of the composite dropped to $15 \mathrm{~W} \mathrm{~m}^{-1}{ }^{\circ} \mathrm{C}^{-1}$ at room temperature. This is due to the fact that $\mathrm{PCBT}$ resin is a poor thermal conductor, and phonon modes within carbon nanotubes can be damped and scattered by the polymer matrix, reducing the thermal conductivity of the carbon nanotubes themselves. ${ }^{[34]}$

Carbon nanotubes are also an excellent conductivity enhancer for nanocomposites due to their high

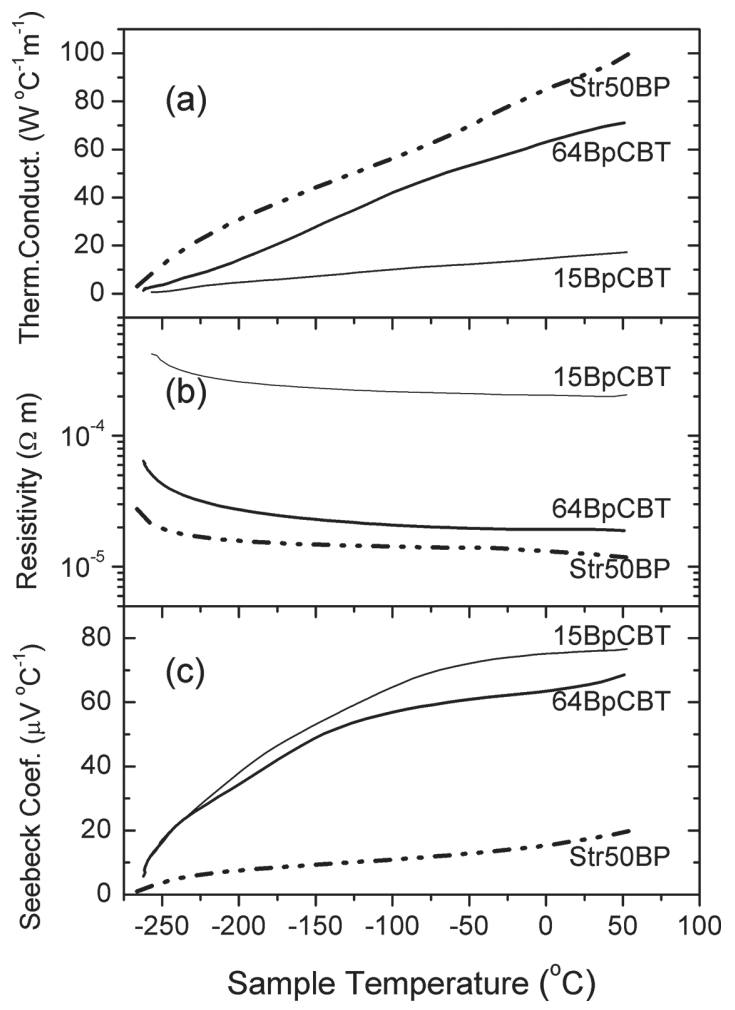

Figure 5. Effect of CNT content on the thermal conductivity (a), the electrical resistivity (b), and the thermal power (c) of buckypaper/pCBT nanocomposites. 
electrical conductivity and high aspect ratio. The dispersion and alignment of carbon nanotubes in the polymer matrix is known to play an important role in directly determining the electrical properties of the polymer composites. ${ }^{[35]}$ Figure $5 \mathrm{~b}$ shows the temperature dependent resistivity of the buckypaper/pCBT composites along with the stretched buckypaper sample. The $50 \%$-stretched buckypaper has very low resistivity of $1.2 \times 10^{-5} \Omega \mathrm{m}$, while pCBT has poor electrical conductivity of approximately $1015 \Omega \mathrm{m}$. The electrical conductivity of the composite was dramatically enhanced by introducing aligned buckypaper. For the composite containing $15 \mathrm{wt} \%$ carbon nanotube (15BPCBT), the resistivity decreased to $2.05 \times 10^{-4} \Omega \mathrm{m}$, approximately 19 orders of magnitude lower than that of the neat pCBT. When 64 wt\% buckypaper was added (64BpCBT), the resistivity dropped to approximately $1.9 \times 10^{-5} \Omega \mathrm{m}$, which was significantly lower than that of composites containing low wt\% CNT content using a regular mixing dispersion approach. ${ }^{[36]}$ These high conductivity measurements may be attributed to carbon nanotube alignment, leading to better contact between the nanotubes.

As shown in Figure $5 \mathrm{c}$, the measured thermoelectric power (TEP, or Seebeck coefficient) values of the buckypaper/pCBT composite samples and the neat 50\%-stretched buckypaper sheets were positive and increased with temperature, characteristic of a moderately $\mathrm{p}$-doped semiconductor, suggesting that holes are the dominant carriers. The p-type characteristics might indicate the formation of new localized acceptor states in the valence band, similar to the situation proposed for X-junction CNTs. ${ }^{[37]}$ The TEP linearly increased at lower temperatures $\left(<-220^{\circ} \mathrm{C}\right)$ for both the buckypaper/ pCBT composites and the neat 50\%-stretched buckypaper sheets. The TEP of the buckypaper/pCBT composite 15BpCBT showed a plateau between $-125^{\circ} \mathrm{C}$ and $-25^{\circ} \mathrm{C}$, and slightly decreased above $-25^{\circ} \mathrm{C}$. At room temperature the Seebeck coefficient of the composites with 15 wt\% buckypaper (15BpCBT) was $75.6 \mu \mathrm{V}{ }^{\circ} \mathrm{C}^{-1}$ and then it decreased with increasing buckypaper content. A Seebeck coefficient value $(S)$ of $64.2 \mu \mathrm{V}^{\circ} \mathrm{C}^{-1}$ was obtained for the composite (64BpCBT) with 64 wt\% buckypaper. The neat 50\%-stretched buckypaper exhibits increasing TEP, even above room temperature. The $S$ values (about $17 \mu \mathrm{V}^{\circ} \mathrm{C}^{-1}$ ) of the 50\%-stretched buckypaper sheets at room temperature were somewhat lower and differed from the linear temperature dependence reported for individual CNTs $\left(S=80 \mu \mathrm{V}{ }^{\circ} \mathrm{C}^{-1}\right.$ at $\left.30{ }^{\circ} \mathrm{C}\right)$ and SWNTs $\left(S=40 \mu \mathrm{V}{ }^{\circ} \mathrm{C}^{-1}\right.$ at $\left.30^{\circ} \mathrm{C}\right),{ }^{[38]}$ which might be caused by interface effects and impurities in our buckypaper sheets.

\subsection{Tensile Performances of the Buckypaper/pCBT Composites as a Function of Buckypaper Content}

The tensile mechanic properties of the resulting buckypaper/pCBT composites were characterized and are listed in Table 1. The stress-strain curves for these composites are displayed in Figure 6. The neat PCBT obtained from the polymerization of $\mathrm{CBT}$ at $250^{\circ} \mathrm{C}$ exhibits a tensile strength of $67 \mathrm{MPa}$ and a Young's modulus of $2.4 \mathrm{GPa}$ with a maximum elongation at break of $3.78 \%$, comparable to commercial PBT. After the in situ polymerization of CBT on $31 \mathrm{wt} \%$ of the $50 \%$-stretched buckyaper, the resulting composite exhibits $361 \mathrm{MPa}$ in strength and $36.9 \mathrm{GPa}$ in modulus, which are approximately 5 times higher and 16-fold higher than those of near PCBT, respectively. Apparently buckypaper works as a suitable reinforcement, involving good interfacial adhesion to $\mathrm{pCBT}$, and accordingly leads to a significant enhancement in the load transfer capability of the composites. The optimized interfacial interaction was achieved at a buckypaper/pCBT weight ratio of around 64:36; the buckypaper/pCBT composite 64BpCBT demonstrated the highest tensile strength of about $600 \mathrm{MPa}$ and Young's modulus of $96 \mathrm{GPa}$, which are the highest performance found in the literature relating to buckypaper/pCBT composites, to the best of our knowledge. Furthermore, on a weight basis, the PCBT composites enhanced with aligned carbon nanotubes offer strong competence over other structural materials such as titanium and

Table 1. The tensile performances of the 50\%-stretched buckypaper/pCBT composites with different nanotube loadings. The mechanical properties of the $50 \%$-stretched buckypaper and the neat $\mathrm{PCBT}$ are also included for comparison.

\begin{tabular}{lcccc}
\hline Sample Name & $\begin{array}{c}\text { Young's Modulus } \\
{[\mathrm{GPa}]}\end{array}$ & $\begin{array}{c}\text { Tensile Strength } \\
{[\mathbf{M P a}]}\end{array}$ & $\begin{array}{c}\text { Elongation at Break } \\
{[\%]}\end{array}$ & $\begin{array}{c}\text { Density } \\
{\left[\mathbf{g} \mathbf{~ c m}^{-3}\right]}\end{array}$ \\
\hline pCBT & $2.4 \pm 0.4$ & $67 \pm 10$ & $3.8 \pm 0.8$ & $1.30 \pm 0.05$ \\
31BpCBT & $36.9 \pm 5.6$ & $361 \pm 54$ & $1.6 \pm 0.5$ & $2.07 \pm 0.05$ \\
44BpCBT & $53.8 \pm 8.1$ & $476 \pm 72$ & $1.4 \pm 0.4$ & $2.04 \pm 0.05$ \\
53BpCBT & $65.4 \pm 9.8$ & $514 \pm 81$ & $1.0 \pm 0.3$ & $1.98 \pm 0.05$ \\
64BpCBT & $95.7 \pm 14.5$ & $598 \pm 90$ & $0.9 \pm 0.2$ & $1.87 \pm 0.05$ \\
Str50Bp & $14.8 \pm 2.3$ & $305 \pm 45$ & $2.8 \pm 0.6$ & $0.86 \pm 0.05$
\end{tabular}

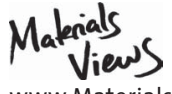




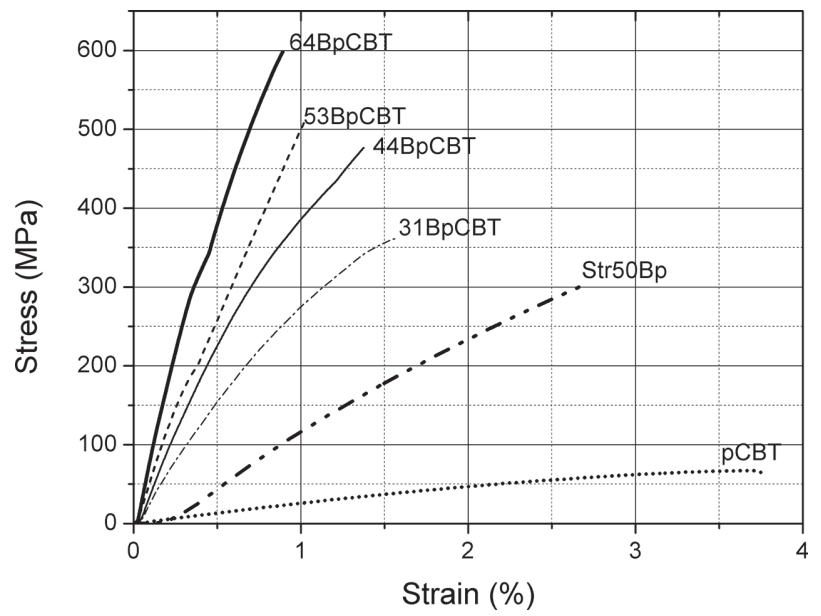

Figure 6. The typical uniaxial tensile stress-strain curves of the 50\%-stretched buckypaper/pCBT composites synthesized using an in situ polymerization approach with different nanotube concentrations. The mechanical properties of the $50 \%$-stretched buckypaper and the neat PCBT are also included for comparison.

aero-grade aluminum, since the density of our composites is below $2.1 \mathrm{~g} \mathrm{~cm}^{-3}$.

Figure 7 shows the cross-sectional fracture SEM image of the $64 \mathrm{BpCBT}$ composites after tensile testing. The pCBT matrix appears to homogeneously cover the aligned CNTs. Good wetting and adhesion of the CNTs with the PCBT are also apparent, indicating a strong interaction and compatibility between the CNTs and the PCBT polymer matrix. This further suggests that our newly developed in situ polymerization technique offers a better dispersion quality of PCBT on buckypaper than the stacking one. The welldispersed lines at the ends of the fractured CNTs suggest adequate load transfer to the CNTs as opposed to pCBT polymer matrix failure and nanotube pull-out characteristics. Moreover, the polymers still remained on the pulledout nanotubes after tensile testing. This kind of nanotube fracture upon tensile testing reflects a strong interfacial adhesion between the CNTs and pCBT matrix, allowing for adequate load transfer from the polymer matrix to the nanotubes. ${ }^{[39]}$ In morphological analysis of the fracture

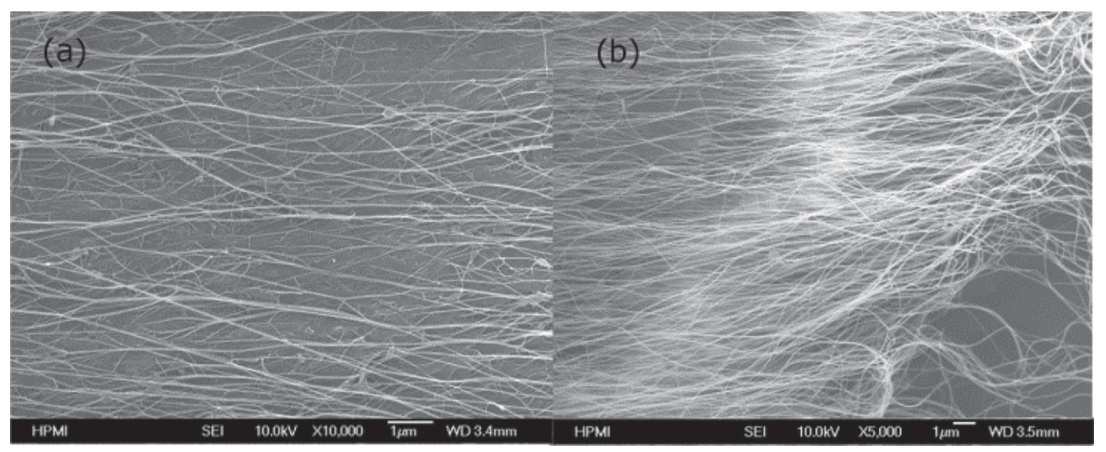

Figure 7. SEM images of the surface morphology (a) and typical fracture (b) of buckypaper/pCBT composites. surfaces of buckypaper/pCBT composites, however, no broken CNTs were observed. This indicates that the load transfer from polymer to CNT was not sufficient to fracture the CNTs. Instead, the failure of the composite appears to arise from pullout of the nanotubes and from mechanical fracture of the pCBT polymer matrix. The presence of a polymer layer on a nanotube after fiber pullout can indicate a strong filler-matrix interface. The actual interfacial energy for a given composite could be estimated by measuring the contact angles between the nanotubes and the polymer.

As can be seen from Figure 8a, the storage moduli of the buckypaper/pCBT composites are basically consistent with the Young's modulus values in the tensile testing (Table 1). The incorporation of buckypaper prominently increased the storage modulus, and the storage moduli of the aligned buckypaper/pCBT composites are much higher than that made from the randomly dispersed CNT buckypaper (rndmBpCBT) at all temperatures. Over most of the temperature ranges tested, the storage modulus increased with increasing nanotube content. A similar phenomenon was observed in graphene/pCBT composites. ${ }^{[7]}$ With 64 wt\% aligned buckypaper loading, the composite sample (64BPmx) exhibits a storage modulus of approximately $84.7 \mathrm{GPa}$ at room temperature while that of the neat PCBT sample is only $2.1 \mathrm{GPa}$. The storage modulus of composites was shown to be strongly dependent on polymer microstructure and crystallinity. ${ }^{[40]}$ This enhanced storage modulus can be understood from the direct contact of the polymer chains with the nanotube surfaces and therefore is influenced by the Van der Waals forces between these aligned tubes and the PCBT matrix. Additionally, another effect might arise from $\pi-\pi$ interactions between the graphene rings of the CNTs and the benzene rings of the terephthalate moiety.

The $\tan \delta$ curve of the PCBT sample exhibits a broad peak around $80^{\circ} \mathrm{C}$, which might correspond to its glass transition temperature $\left(T_{\mathrm{g}}\right)$. The glass transition temperature is affected by the mobility of polymer chains. An apparent upshift of the glass transition temperature was observed with increasing buckypaper content. The increase in $T_{\mathrm{g}}$ might be associated with high filling and efficient reinforcement, that is to say, favorable interactions between the PCBT matrix and the CNTs restricted the mobility of the polymeric chains. Interestingly, it seems that the glass transition temperature is not affected very much by the orientation of nanotubes in the buckypaper, but the buckypaper loading. This finding is in line with the reinforcing action of CNTs, and also suggests the formation of an interphase 

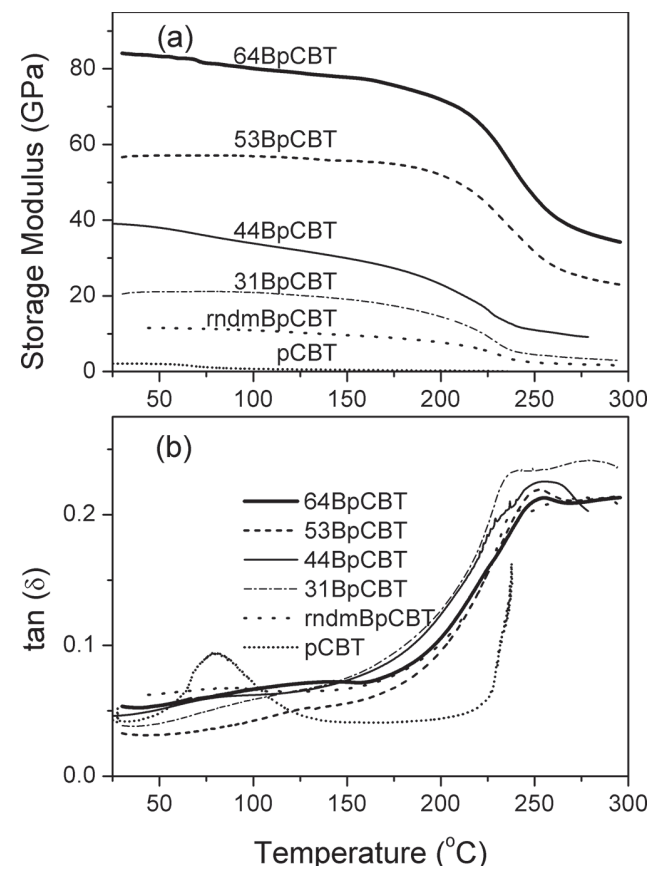

Figure 8. The dynamical mechanic analysis of aligned buckypaper/ pCBT composites as a function of buckypaper content: a) Comparisons of storage modulus; b) Comparisons of $\tan \delta$ curves of buckypaper/pCBT composites. The composite (rndmBpCBT) of randomly dispersed CNT buckypaper (ca. $70 \mathrm{wt} \%$ ) and polymerized CBT (pCBT) are also included for comparison.

between CNTs and PCBT (possibly transcrystalline-like) in which the segmental motion of PCBT macromolecules is restricted in the presence of $\mathrm{CNTs} .{ }^{[41]}$

Compared with the buckypaper/pCBT composites through stacking approach, ${ }^{[42]}$ our in situ polymerized composites exhibit better mechanical performance (Table S1 in the Supporting Information). This can be understood from the fact that solution-based polymerization can guarantee uniform dispersion of CBT molecules on the wall surfaces of CNTs (Figure S2 in the Supporting Information), as a consequence leading to better interfacial interaction between PCBT and CNT.

\section{Conclusion}

Ultimately, we have developed in situ polymerization approach for the synthesis of high performance buckypaper/pCBT thermoplastic composites. The resulting composites show several times better tensile performance than the neat component, i.e., pCBT and buckypaper. For the buckypaper/pCBT composite containing 64 wt\% of $50 \%$-stretched buckypaper, the tensile strength and modulus reached nearly $600 \mathrm{MPa}$ and $96 \mathrm{GPa}$, respectively. To the best of our knowledge, these are highest tensile properties reported in the literature. The in-situ polymerized buckypaper/pCBT composites also exhibit better tensile performance than their counterparts fabricated through a stacking method. This may be explained as follows. 1) The solution approach guarantees better dispersion of CBT oligomers on the network of buckypaper. 2) Aligned tubes are used as a framework for the composite. Along the alignment direction, the mechanical properties were significantly improved due to the increased carbon nanotube rope sizes and the higher packing density compared to pre-stretched buckypaper. 3) In situ polymerization can facilitate the interfacial interaction between the pCBT chains and nanotubes. It is worth mentioning that the performance of the buckypaper/pCBT composites can be further improved by employing long CNTs and aligning the pCBT chain along the direction of the aligned CNTs. Additionally, our approach to fabricating high performance buckypaper/pCBT composites can be easily scaled up for numerous real industrial applications, such as for aircraft, rockets, vehicles, and so on.

\section{Supporting Information}

Supporting Information is available from the Wiley Online Library or from the author.

Acknowledgements: This research was supported by the ONR (N00014-11-1-0274) project. Sponsorship and oversight of the program by Dr. Ignacio Perez are greatly appreciated. The thermal and electrical property measurements were conducted at the National High Magnetic Field Laboratory with the assistance of Dr. Jun Lu.

Received: August 26, 2014; Revised: October 7, 2014; Published online: November 10, 2014; DOI: 10.1002/macp.201400443

Keywords: carbon nanotubes; composites; cyclic butylene terephthalate; in situ polymerization; thermoplastics

[1] A. R. Tripathy, W. Chen, S. N. Kukureka, W. J. MacKnight, Polymer 2003, 44, 1835.

[2] H. Parton, I. Verpoest, Polym. Compos. 2005, 26, 60.

[3] A. R. Tripathy, E. Burgaz, S. N. Kukureka, W. J. MacKnight, Macromolecules 2003, 36, 8593.

[4] A. R. Tripathy, A. Elmoumni, H. H. Winter, W. J. MacKnight, Macromolecules 2005, 38, 709.

[5] G. Romhany, J. Vigh, R. Thomann, J. Karger-Kocsis, I. E. Sajo, Macromol. Mater. Eng. 2011, 296, 544.

[6] J. Karger-Kocsis, T. Bárány, Compos. Sci. Techn. 2014, 92, 77.

[7] G. Balogh, S. Hajba, J. Karger-Kocsis, T. Czigany, J. Mater. Sci. 2013, 48, 2530.

[8] Carbon Nanotubes: Synthesis, Structure, Properties, and Applications, (Eds: M. S. Dresselhaus, G. Dresselhaus, P. Avouris), Springer, New York, USA 2001.

[9] a) J. A. Semlyen, E. R. Semlyen, Cyclic Polymers, Springer, New York, USA, 2001; b) T. Czigány, J. Karger-Kocsis in Handbook of Thermoplastic Polyesters: Homopolymers, Copolymers, Blends and Composites (Ed: S. Fakirov), Wiley-VCH, Weinheim, Germany, 2002, pp. 1133-1171.

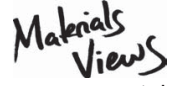

www.MaterialsViews.com 
[10] Z. A. Mohd Ishak, K. G. Gatos, Karger-Kocsis, J. Polym. Eng. Sci. 2006, 46, 743.

[11] T. Yu, C. M. Wu, C. Y. Chang, C. Y. Wang, S. P. Rwei, Expr. Polymer Lett. 2012, 6, 318.

[12] T. Abt, A. Martínez de Ilarduya, J. J. Bou, M. Sánchez-Soto, Expr. Polymer Lett. 2013, 7, 172.

[13] C.-S. Park, K.-J. Lee, S. W. Kim, Y. K. Lee, J.-D. Nam, J. Appl. Polym. Sci 2002, 86, 478.

[14] H.-J. Radusch, in Handbook of Thermoplastic Polyesters: Homopolymers, Copolymers, Blends and Composites, (Ed: S. Fakirov), Wiley-VCH, Weinheim, Germany, 2002, pp. 389-419.

[15] M. Harsch, J. Karger-Kocsis, A. A. Apostolov, J. Appl. Polym. Sci. 2008, 108, 1455.

[16] G. Romhany, J. Vigh, R. Thomann, J. Karger-Kocsis, I. E. Sajo, Macromal. Mater. Eng. 2011, 296, 544.

[17] H. Koerner, W. D. Liu, M. Alexander, P. Mitau, H. Dowty, R. A. Vaia, Polymer 2005, 46, 4405.

[18] A. I. Balabanovich, T. A. Zevaco, W. Schnabel, Macromol. Mater. Eng. 2004, 289, 181.

[19] M. Pellow-Jarman, M. Hetem, Polym. Degrad. Stabil. 1995, 47, 413.

[20] O. F. Solomon, I. Z. Ciuta, J. Appl. Polym. Sci. 1992, 6, 683.

[21] F. Wu, G. Yang, J. Appl. Polym. Sci. 2010, 118, 2929.

[22] Z. A. Mohd Ishak, P. P. Shang, J. Karger-Kocsis, J. Thermal Anal. Calorim. 2006, 84, 637.

[23] D. C. Bassett, R. H. Olley, I. A. M. Raheil, Polymer 1988, 29, 1745.

[24] M. C. Righetti, M. Pizzoli, N. Lotti, A. Munari, Macromol Chem. Phys. 1998, 199, 2063.

[25] H. J. Lee, S. J. Oh, J. Y. Choi, J. W. Kim, J. Han, L. S. Tan, J. B. Baek, Chem. Mater. 2005, 17, 5057.
[26] T. M. Wu, E. C. Chen, J. Polym. Sci., Part B: Polym. Phys. 2006, 44, 598.

[27] P. Kim, L. Shi, A. Majumdar, P. L. McEuen, Phys. Rev. Lett. 2001, 87, 215502.

[28] C. W. Nan, G. Liu, Y. Lin, M. Li, Appl. Phys. Lett. 2004, 85, 3549.

[29] H. Huang, C. Liu, Y. Wu, S. Fan, Adv. Mater. 2005, 17, 1652.

[30] J. Hone, M. Whitney, C. Piskoti, A. Zettl, Phys. Rev. B 1999, 59, 2514.

[31] J. Hone, M. C. Llaguno, N. M. Nemes, A. T. Johnson, J. E. Fischer, D. A. Walters, M. J. Casavant, J. Schmidt, R. E. Smalley, Appl. Phys. Lett. 2000, 77, 666.

[32] G. W. C. Kaye, T. H. Laby, Tables of Physical and Chemical Constants, 16th ed., Longman, London, UK 1995.

[33] J. G. Park, O. Cheng, J. Lu, J. Bao, S. Li, Y. Tian, Z. Liang, C. Zhang, B. Wang, Carbon 2012, 50, 2083.

[34] Y. Mamunya, A. Boudenne, N. Lebovka, L. Ibos, Y. Candau, M. Lisunova, Compos. Sci. Technol. 2008, 68, 1981.

[35] M. S. P. Shaffer, A. H. Windle, Adv. Mater. 1999, 11, 937.

[36] M. Mukherjee, T. Das, R. Rajasekar, S. Bose, S. Kumar, C. K. Das, Compos. Part. A 2009, 40, 1291.

[37] J.-C. Charlier, Acc. Chem. Res. 2002, 35, 1063.

[38] C. Yu, L. Shi, Z. Yao, D. Li, A. Majumdar, Nano Lett. 2005, 5, 1842.

[39] N. G. Sahoo, H. K. F. Cheng, L. Li, S. H. Chan, J. Zhao, Adv. Funct. Mater. 2009, 19, 3962.

[40] N. G. Sahoo, H. K. F. Cheng, J. Cai, L. Li, S. H. Chen, J. Zhao, S. Yu, Mater. Chem. Phys. 2009, 117, 313.

[41] C. McClory, T. McNally, G. P. Brennan, J. Erskine, J. Appl. Polym. Sci. 2007, 105, 1003.

[42] C.-Y. Chang, E. M. Phillips, R. Liang, S. W. Tozer, B. Wang, C. Zhang, H.-T. Chiu, J. Appl. Polym. Sci. 2013, 128, 1360. 